\title{
Designing Control Software for Vehicle-to-Vehicle Communication Device Applied to Model-Based Design Technique
}

\author{
Jaewoo Yoon ${ }^{1}$ and Byeongwoo $\mathrm{Kim}^{2 *}$ \\ ${ }^{1,2}$ Dept. of Electrical Engineering, University of Ulsan \\ ${ }^{1}$ jewos0127@gmail.com, ${ }^{2 *}$ bywokim@ulsan.ac.kr
}

\begin{abstract}
Recently, Advanced Driver Assistance System (ADAS) technology has been in development to improve drivers' convenience and safety. Research has been actively conducted on technology that improves safety by viewing vehicles as single sensors and using communications between vehicles to share information. Wireless Access in Vehicular Environment (WAVE) and Continuous Access for Land Mobiles (CALM) are two of the most commonly used vehicle-to-vehicle communication technologies; this study uses WAVE. The existing logical design of software has the drawback of consuming too much time and money for development as the software engineer and algorithm developer work independent of each other. Therefore, this study proposes a method to design control software for a WAVE communication device by applying the Model-Based Design (MBD) technique, which meets the ISO 26262 standard, and performs verification using MicroAutoBox II (MABX-II), a Rapid Control Prototyping (RCP) device made by dSPACE.
\end{abstract}

Keywords: Advanced Driver Assistance System, Wireless Access in Vehicular Environment, Model-Based Design, Rapid Control Prototyping, Basic Safety Message

\section{Introduction}

Recently, studies on Advanced Driver Assistance System (ADAS) technology development have been actively conducted to improve drivers' convenience and safety. Of all the different ADAS systems, Autonomous Emergency Braking (AEB) prevents potential collisions by using information obtained from various sensors (radar, lidar, camera, etc.) attached to the vehicle [1, 2]. The Euro New Car Assessment Programme (NCAP), an institution that determines the safety rating of European vehicles, published its report on AEB and started including the system in its safety ratings beginning in 2014. It also published the "Euro NCAP 2020 Roadmap," expanding the function beyond vehicle-to-vehicle to include vehicle-to-Vulnerable Road Users (VRUs) such as pedestrians and bicycles[3]. Furthermore, studies have been conducted to improve safety by adjusting the point of using the brakes of the AEB system based on road gradients and conditions $[4,5]$.

However, the vehicle-mounted sensor-based AEB system is not fully operational for blind spots created by obstacles, crossroads, or curved roads because of limits in the sensors' measurement range. To overcome this limitation, research studies are being actively conducted to remove such blind spots and increase the reliability of the AEB system by viewing vehicles as single sensors and by using vehicle-to-vehicle communications [6-9]. For example, research is being carried out to detect other vehicles in blind spots so the AEB system can operate fully even when the vehicle enters a curved road where the sensor's detection range cannot reach [8], or a crossroad in a downtown

Received (January 4, 2018), Review Result (March 8, 2018), Accepted (March 12, 2018)

${ }^{*}$ Corresponding Author 
area full of high-rise buildings [9]. In addition, the US National Highway Traffic Safety Administration (NHTSA) published a report on a technology that increases safety by preventing accidents through a Vehicle-to-Vehicle (V2V) method [10]. For stable operations of such vehicle safety services, a smooth communication environment is required even in different situations where vehicles are moving at high speed.

The two most typical vehicle-to-vehicle communications are Wireless Access in Vehicular Environment (WAVE) and Continuous Access for Land Mobiles (CALM). WAVE uses 5.9-GHz frequency band based on the IEEE communication standards and is composed of IEEE 802.11p and IEEE 1609.x standards. IEEE 802.11p includes physical and MAC layers, IEEE 1609.1 the ITS application service layer, IEEE 1609.2 the certification and security layers, IEEE 1609.3 the network layer, and IEEE 1609.4 the multichannel layer. In addition, CALM is a technology defined in WG16, one of the subgroups of ISO TC204, a pioneer in the standardization of the ITS field. CALM provides a vehicle-to-vehicle communication environment through cellular and artificial satellites $[11,12]$.



\section{Figure 1. WAVE Communication Device: External Device Connection Diagram}

With the rapid electronization of vehicles, the complexity of automotive electronic control systems is also quickly increasing. In this context, designing control systems with the existing traditional method is exposing limitations in the system design, implementation, and verification process. In addition, accidents occurring from errors owing to increased system complexity are on the rise. To prevent these issues, the International Standards Organization (ISO) established ISO 26262, an international standard for the functional safety of automobiles, and has legal regulations in place. ISO 26262 consists of a total of 10 parts as well as 43 requirements and recommendations, and the V-Model development process is applied to both hardware and software. The VModel development process follows the Model-Based Design (MBD) technique[13]-[15]. This process satisfies various and complex requirements and also guarantees the design of a safe system that runs without errors. Furthermore, it shortens the development period considerably by automatically supporting the verification of software and hardware through Model-in-the-Loop (MIL), Software-in-the-Loop (SIL), and Hardware-in-theLoop (HIL) testing.

This study proposes a method of designing control software for a WAVE communication device using the MBD technique, and performs a verification of the control software using MicroAutoBox II, a Rapid Control Prototyping (RCP) equipment made by dSPACE. 


\section{System Design}

\subsection{Analysis of Logical Structure of Software}

This study uses an eight-band antenna developed by INFAC and a WAVE communication device developed by Korea Electronics Technology Institute (KETI). The WAVE communication device can be connected to external devices via ISP, UART, and Ethernet (UDP/IP), and performs data communication with either Road-Side Equipment (RSE) or On-Board Equipment (OBE). The WAVE communication device can register/deregister the WAVE Short Message (WSM) service and transmit/receive WSM frames. The external device connected to the WAVE communication device is designed to act as a Higher Layer, while the WAVE communication device is designed to act as the Networking Service and the MAC/PHY layers.

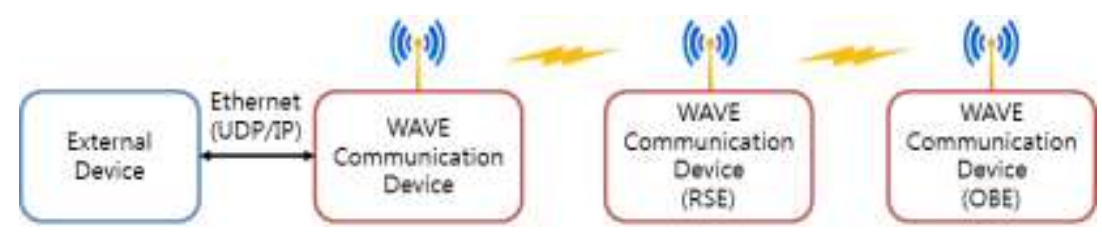

Figure 2. WAVE Communication Device: External Device Connection Diagram

Octets \begin{tabular}{c|c|c|c|c|c|}
14 & 20 & 8 & 2 & 1 & var. \\
\begin{tabular}{|c|c|c|c|} 
MAC \\
Header
\end{tabular} & $\begin{array}{c}\text { IP } \\
\text { Header }\end{array}$ & $\begin{array}{c}\text { UDP } \\
\text { Header }\end{array}$ & $\begin{array}{c}\text { Seq. } \\
\text { Number }\end{array}$ & $\begin{array}{c}\text { Msg. } \\
\text { Type }\end{array}$ & Msg. \\
\hline
\end{tabular}

Figure 3. Basic Message Format of WAVE Communication Device

The basic message format of the WAVE communication device consists of a MAC Header, IP Header, UDP Header, Sequence Number, Message Type, and Message. The sequence number increases by 1 every time a request message is sent for confirmation of service or management information. When the response message is sent, the sequence number stored in the request message must be included in the response. The response message and the message type provided by the WAVE communication module used in this study are as follows:

Table 1. Response Message of WAVE Communication Module

\begin{tabular}{|l|}
\hline Response Message \\
\hline $0 \times 00$ \\
\hline $0 \times 01$ \\
\hline
\end{tabular}

Table 2. Message Type of WAVE Communication Module

\begin{tabular}{|l|}
\hline Message Type \\
\hline WSM Service Register \\
\hline WSM Service Deregister \\
\hline WSM Service Reply \\
\hline MGMT Get Request \\
\hline MGMT Get Reply \\
\hline MGMT Set Request \\
\hline WSM Data \\
\hline WSM Data Reply \\
\hline Connection Request \\
\hline
\end{tabular}




\subsection{Achieving MBD-based Modularization and its Evaluation}

\subsubsection{Composition of Control Software System for WAVE Communication Module}

The control logic for the WAVE communication device was designed using MATLAB/Simulink based on the MBD development method. The control logic consists of a Controller, ETHERNET_UDP_RX, and ETHERNET_UDP_TX. An RTI ETHERNET Blockset made with dSPACE was employed to use the UDP/IP of the RCP equipment.

The Controller is composed of six subsystems: Upper, RcvControl, SndControl, DistributeRcvData\&DataSets, WsmDataHandling, and MgmtDataHandling. Based on the logical structure of the software analyzed above, the Controller generates and transmits a signal that controls the WAVE communication device, and also receives and processes the feedback and the WSM data from the WAVE communication device.

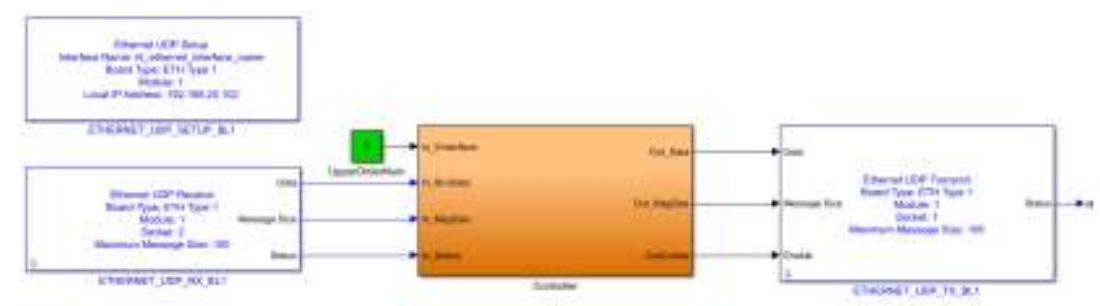

Figure 3. Design of Control S/W Model of WAVE Communication Device

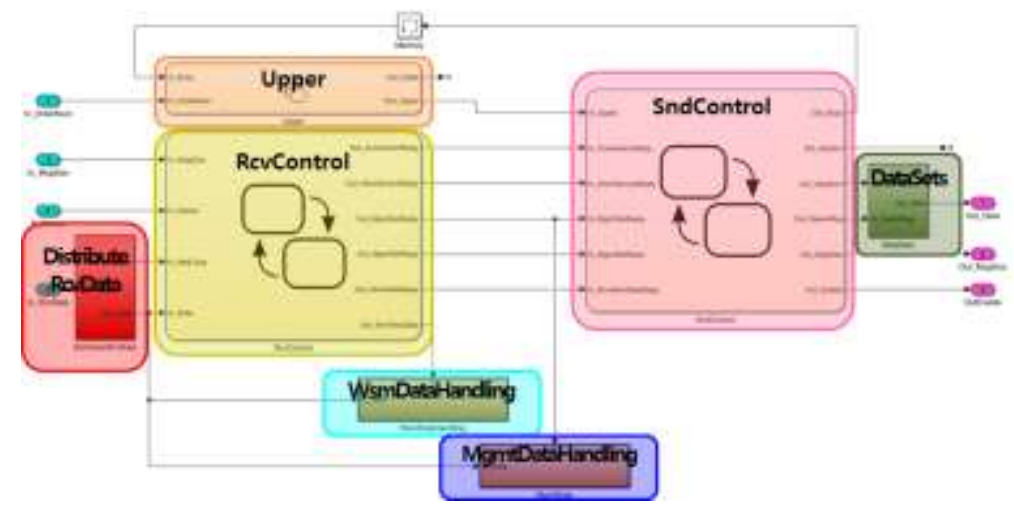

Figure 4. Components of Controller Block

\subsubsection{Achieving MBD-based Modularization for WAVE Communication Module}

\subsubsection{Upper Block}

The Upper Block controls the SndControl Block based on the control command (WSM service re/deregister, MGMT get/set, WSM data, etc.) from the user (or the highest controller).

\subsubsection{SndControl Block}

Once the power goes on, the WAVE communication device is configured to resend a "Connection Request" every second until it receives a "Connection Reply" containing the IP address and the port number of the external device. Upon receipt of the "Connection Reply," it is designed to transmit messages such as WSM service Re/Deregister and MGMT Get/Set. 


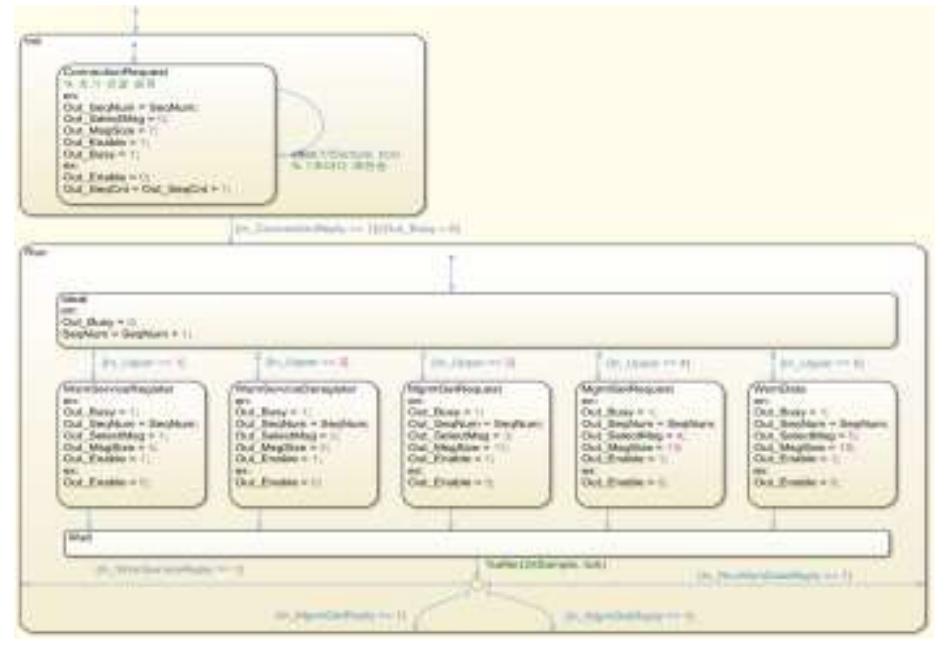

Figure 5. SndControl Block Configuration

\subsubsection{RevControl Block}

The RcvControl Block activates either the WsmDataHandling Block or the MgmtDataHandling Block based on the Msg Type of the message received from the WAVE communication device. It transmits a Reply signal to SndControl so the next command can be sent.

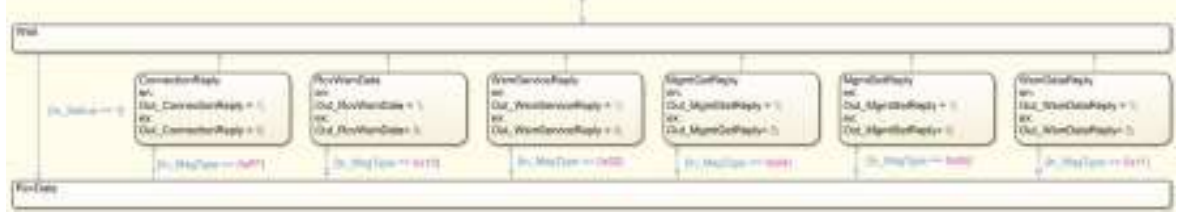

Figure 6. RcvControl Block Configuration

\subsubsection{DataSets and DistributeRcvData Block}

As a block that converts the format to transmit messages from external devices to the WAVE communication device, the DataSets \& DistributeRcvData Block classifies the format according to Seq Num, Msg Type, and Msg, and then relays the message to either the RcvControl Block or the WAVE communication device.

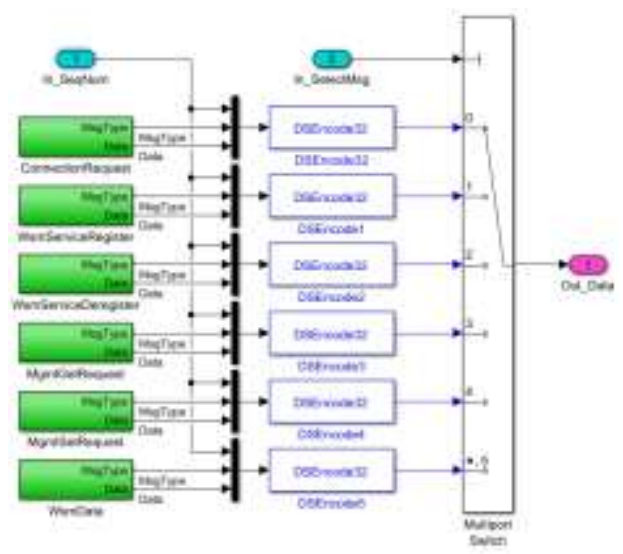

Figure 7. DataSets Block Configuration 


\subsubsection{MgmtDataHandling Block}

When the Msg Type received from the WAVE communication device is "MGMT Get Reply," the MgmtDataHandling Block is activated to extract the situation information of the WAVE communication device based on the Element ID (power, data rate, channel, etc.,).

\subsubsection{WsmDataHandling Block}

When the Msg Type received from the WAVE communication device is "WSM Data," the WsmDataHandling Block is activated to extract WSM Data and situation information such as the Peer Mac Address, WSMP Version, PSID, and WSM Length. It is also designed to extract DSRC Message information (ID, count, temporary ID, etc.), GPS information (latitude, longitude, altitude, speed, heading angle, acceleration per axis, etc.) and vehicle information (steering wheel angle, brake system condition, gear condition, vehicle size, etc.,) when the WSM Data receives SAE_J2735 BSM.

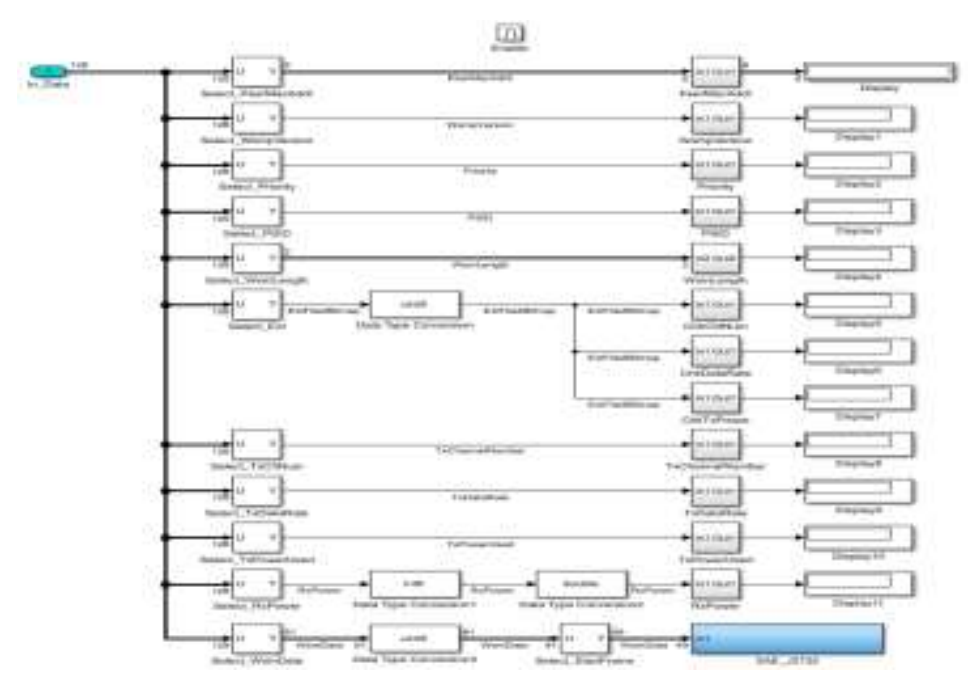

Figure 8. WsmDataHandling Block Configuration

\subsubsection{Evaluation of Control Software for WAVE Communication Module}

An RCP device was used to evaluate the control software for the WAVE communication module. Using RCP equipment for software evaluation has the advantages of enabling easy application of increasingly complex automotive electronic control systems software, rapid solutions for occurring defects, and improvements in performance through code verification.

This study used MicroAutoBox II (MABX-II), an RCP device made by dSPACE. One of its strengths is that it can immediately port the control logic designed through MATLAB/Simulink. It can also monitor the parameters and situation information of the control logic in real time by using ControlDesk made by dSPACE. A module developed by KETI and an eight-band antenna produced by INFAC were used for the WAVE communication device. The device was set up to run in debug mode, and it was checked to see that messages were being properly transmitted and received through the terminal. In addition, BSM was transmitted using MK5-OBU made by CohdaWireless, and all WSMs transmitted and received by specific channels were monitored by running the Sniffer/Monitor mode. Moreover, Wireshark, a packet analysis tool, was configured to confirm the information of sent and received messages in real time. 


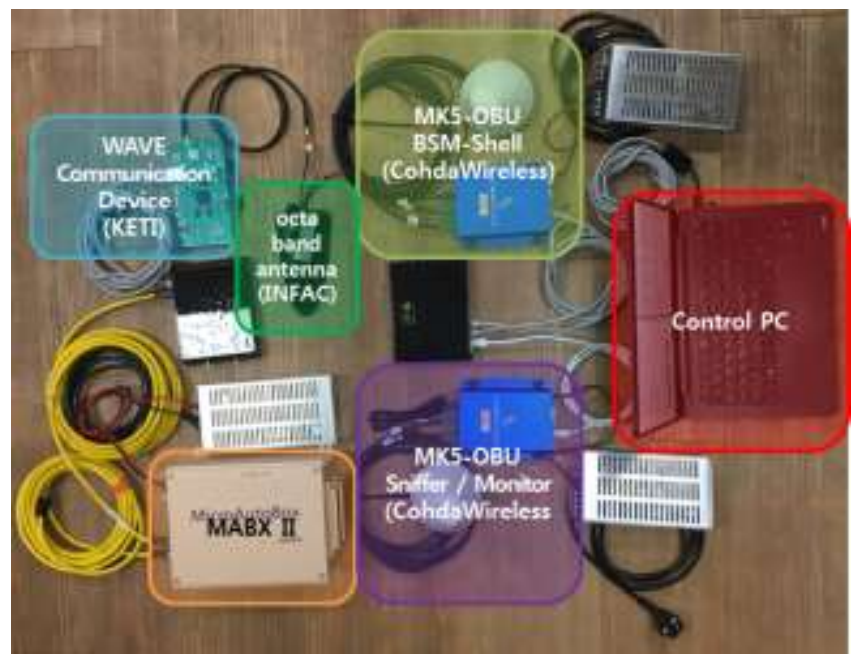

Figure 9. Configuration of Verification Environment
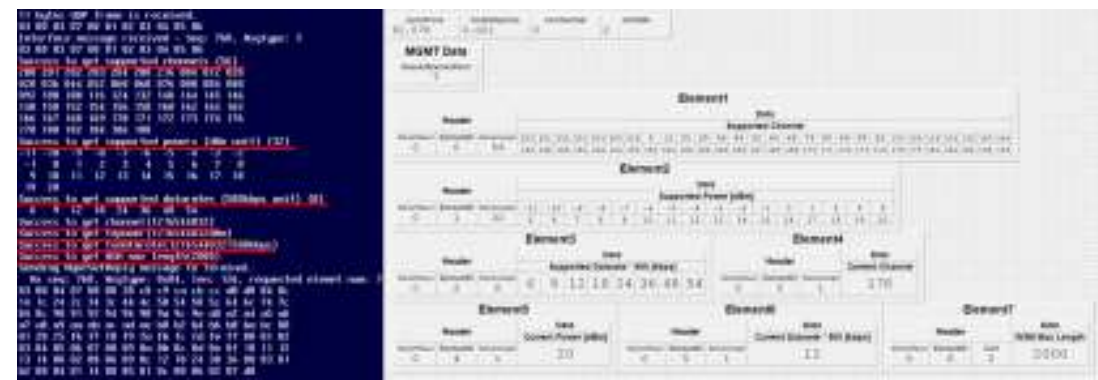

Figure 10. Result of "MGMT Get Reply"
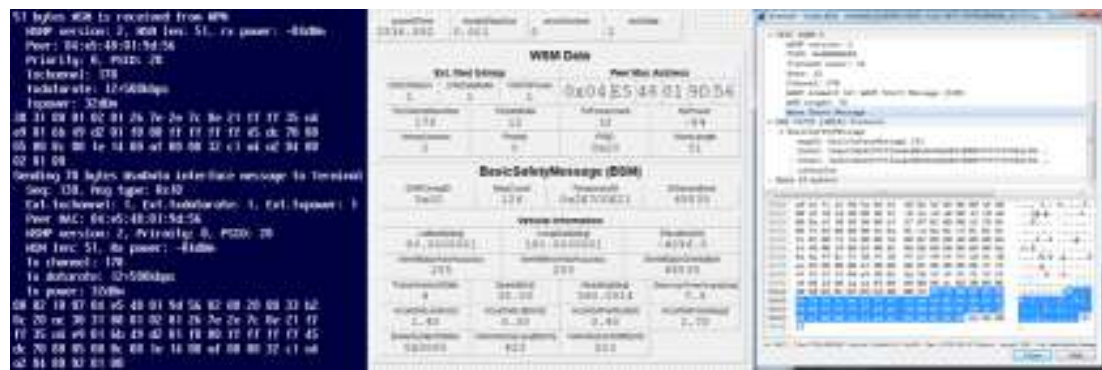

Figure 11. Result of "WSM Data (BSM)" (Left: Terminal, Center: ControlDesk, Right: wireshark)

When the power went on, the MABX-II IP address and port number were transmitted using the "Connection Request" message, and the "Connection Reply" message was received properly from the WAVE communication device. It was also confirmed that "MGMT Get Request" was transmitted to receive and extract the situation information of the WAVE communication device based on the Element ID.

Furthermore, it was confirmed that the WAVE communication device received BSM [DSRC Message information (ID, count, temporary ID, etc.), GPS information (latitude, longitude, altitude, speed, heading angle, acceleration per axis, etc.,) and vehicle information (steering wheel angle, brake system condition, gear condition, vehicle size, etc.,)] transmitted from MK5-OBU, and sent it properly in the form of a "WSM Data" message to MABX-II. Here, the Provider Service Identifier (PSID) was set to 0x20 to receive $\mathrm{BSM}$. 


\section{Conclusion}

Studies on ADAS are being conducted to improve driver safety and convenience, and studies specifically on technology that views vehicles as single sensors and uses $\mathrm{V} 2 \mathrm{~V}$ to share information to prevent accidents are underway. A WAVE communication device that satisfies IEEE $802.11 \mathrm{p}$ was used as a V2V communication device. The module for the WAVE communication device was developed by KETI and the octaband antenna by INFAC. In addition, to prevent accidents arising from errors owing to the increased complexity of the control logic, this study designed a control logic for the WAVE communication device using the MBD technique, a V-Model development process that satisfies ISO 26262. Furthermore, MABX II, an RCP device made by dSPACE, was used to verify the designed control logic and to confirm that various message transmissions/receptions and WSM data (BSM) receptions were all working correctly. In the future, not only BSM but also other J2735 messages will be analyzed and applied.

\section{Acknowledgement}

This research was supported by the MSIT(Ministry of Science and ICT), Korea, under the ITRC(Information Technology Research Center) support program(IITP-2017-2012-000628) supervised by the IITP(Institute for Information \& communications Technology Promotion) and a Future Automobile Parts Technology Development Project supported by Ulsan Metropolitan City and Ulsan Technopark, "Autonomous driving/connected car production and demonstration business" and this paper is a revised and expanded version of a paper entitled "Design of Control Software for WAVE Communication Module" presented at "The $1^{\text {st }}$ International Conference on Convergent Research Theory and Technology (CRTT) 2017”.

\section{References}

[1] W. Hulshof, I. Knight, A. Edwards, M. Avery and C. Grover, "Autonomous emergency braking test results", Proc. 23rd International Technical Conf. Enhanced Safety of Vehicles (ESV), (2013).

[2] Y. Woo and I. Kim, "Alarm System for Pedestrians Safety Against Hybrid/PHEV/EV Vehicle on Minor Road”, International Journal of u-and e-Service, Science and Technology, vol. 9, no. 7, (2016), pp. 361368.

[3] N. C. A. P. Euro, "2020 Roadmap, Revision 1”, Available from: http://www. Euroncap.com, (2015).

[4] M. Lin, J. Yoon and B. Kim, "A Study on Emergency Braking System Algorithm that Considers Changes in Incline Angles in Real Time", International Journal of Applied Engineering Research (IJAER), vol. 11, no. 2, (2016), pp. 1099-1103.

[5] M. Lin, J. Yoon and B. Kim, "A Study and Analysis for Calculating the Brake-application Time of AEB Systems Considering the Gradient", International Journal of Control and Automation (IJCA), vol. 8, no. 6, (2015), pp. 283-292.

[6] H. Hartenstein and K. P. Laberteaux, "A Tutorial Survey on Vehicular Ad Hoc Networks", IEEE Communications Magazine, vol. 46, no. 6, (2008), pp. 164-171.

[7] S. Jeon, J. Lee and B. Kim, "A Study on Performance Analysis of V2V Communication Based AEB System Considering Road Friction at Slopes", International Journal of Future Generation Communication and Networking (IJFGC), vol. 9, no. 11, (2016), pp. 71-80.

[8] H. Jo and B. Kim, "Performance Improvement of Collision Warning System on Curved Road Based on Intervehicle Communication", Mathematical Problems in Engineering, vol. 2015, Article ID 838929, (2015).

[9] S. Jeon, G. Kim and B. Kim, "Braking Performance Improvement Method for V2V Communicationbased AEB System at Various Intersections", International Journal of Applied Engineering Research (IJAER), vol. 10, no. 19, (2015), pp. 40005-40009.

[10] J. Harding, G. Powell, R. Yoon, J. Fikentscher, C. Doyle, D. Sade, M. Lukuc, J. Simons and J. Wang, "Vehicle-to-vehicle communications: Readiness of V2V technology for application", National Highway Traffic Safety Administration (NHTSA), DOT HS 812 014, (2014).

[11] S. Kim, "A Study on The Frequency Allocation of WAVE for Smart Vehicle Industry", Journal of the Institute of Electronics and Information Engineers, vol. 51, no. 6, (2014), pp. 183-189.

[12] S. Gao, A. Lim and D. Bevly, "An Empirical Study of DSRC V2V Performance in Truck Platooning Scenario", Digital Communications and Networks, vol. 2, no. 4, (2016), pp. 233-244. 
[13] M. Broy, S. Kirstan, H. Krcmar, B. Schätz and J. Zimmermann, "What is the benefit of a model-based design of embedded software systems in the car industry?", Software Design and Development: Concepts, Methodologies, Tools, and Applications: Concepts, Methodologies, Tools, and Applications, (2013), pp. 310-334.

[14] T. Lee, I. Yoo, B. Kim, G. Kim and J. Hur, "A Study on Power Characteristic of Electric Motorcycle using Automotive Simulation Models", SAE Technical Paper, No. 2013-01-0059, (2013).

[15] X. Liu, "Drive Control Strategy Simulation Research of Plug-in Hybrid Electric Vehicle Based on Matlab/Simulink Platform", International Journal of u-and e-Service, Science and Technology, vol. 9, no. 3, pp. 43-52, (2016).

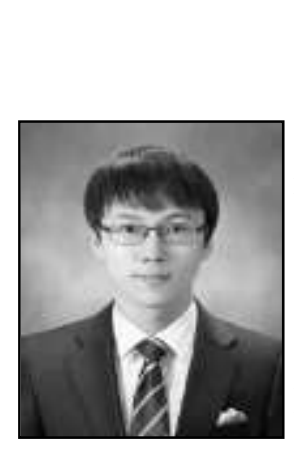

\section{Authors}

Jaewoo Yoon, Received the B.E degree in electrical engineering in University of Ulsan, Ulsan, South Korea. He is pursuing his M.E degree in electrical engineering in University of Ulsan, Ulsan, South Korea. His current research interests include advanced driving assistance system (ADAS), autonomous emergency braking (AEB) system, information fusion theories and application for vehicle localization, and design of intelligent vehicles.

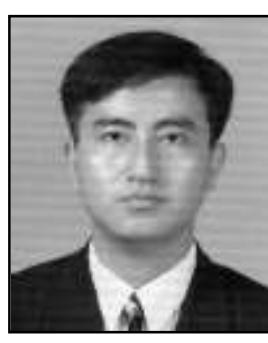

Byeongwoo Kim, Received the B.E, M.E. and Ph.D degree in Precision Mechanical Engineering from Hanyang University. He worked at KOSAKA Research Center in 1989. He worked at KATECH electrical technology Research Center from 1994 to 2006. Now he is a professor in the School of electrical engineering in University if Ulsan, Ulsan, South Korea from 2006. His current research interests include advanced driving assistance system (ADAS), and autonomous emergency braking (AEB) system. 
International Journal of Control and Automation

Vol. 11, No. 7 (2018) 